curtain contamination occurred predominantly in rooms with preexisting patient colonization, often with matching isolates.

Privacy curtains serve an important role in healthcare settings, but they are a potential pathogen reservoir due to large surface area and frequent contact by HCWs and patients. ${ }^{1-4,6}$ Prior studies in intensive care units have reported rapid MRSA or VRE contamination, within 1 week of new curtain placement. ${ }^{4,5}$ Transmission of bacteria to HCW hands occurs after $50 \%$ of curtain contacts. ${ }^{10}$ Our study showed the more common sequence of events to be patient colonization followed by curtain contamination; however, in some cases in which curtain contamination preceded the patient colonization, the curtain was a plausible source of transmission to patients.

In this hypothesis-generating study, we did not assess the directionality of MDRO contamination or quantify the level of curtain contamination. Curtain cleaning practices, optimal frequency of cleaning, and any potential transmission to roommates should be investigated in-depth. To our knowledge, this is the first investigation of curtain contamination in nursing home settings, in which the prevalence of MDRO patient colonization is particularly high. Widespread curtain contamination has been linked to lack of regular disinfection or replacement. ${ }^{6}$ Antimicrobial curtains, including antimicrobial textile technology, have been studied as a potential solution. ${ }^{5}$

Action can and should be taken to decrease curtain contamination, including standard practice guidelines for cleaning or replacing curtains, and implementation of simple strategies, such as better handwashing by medical staff, patients, and visitors, while also considering alternative designs, such as removable handles, retractable partitions, and argon glass doors.

Acknowledgments. We thank all nursing home patients and HCWs who participated in this research study.

Financial support. This work was supported by the National Institutes of Health (grant nos. RO1 AG041780 and K24 AG050685).
Conflicts of interest. All authors report no conflicts of interest relevant to this article.

Supplementary material. To view supplementary material for this article, please visit https://doi.org/10.1017/ice.2021.60

\section{References}

1. Fijan S, Turk SS. Hospital textiles, are they a possible vehicle for healthcare-associated infections? Int J Environ Res Public Health 2012;9: 3330-3343.

2. Mitchell BG, Dancer SJ, Anderson M, Dehn E. Risk of organism acquisition from prior room occupants: a systematic review and meta-analysis. J Hosp Infect 2015;91:211-217.

3. Shek K, Patidar R, Kohja Z, et al. Rate of contamination of hospital privacy curtains in a burns/plastic ward: a longitudinal study. Am J Infect Control 2018;46:1019-1021.

4. Ohl M, Schweizer M, Graham M, Heilmann K, Boyken L, Diekema D. Hospital privacy curtains are frequently and rapidly contaminated with potentially pathogenic bacteria. Am J Infect Control 2012;40: 904-906.

5. Schweizer M, Graham M, Ohl M, Heilmann K, Boyken L, Diekema D. Novel hospital curtains with antimicrobial properties: a rando mized, controlled trial. Infect Control Hosp Epidemiol 2012;33: 1081-1085.

6. Sehulster LM. Healthcare laundry and textiles in the United States: review and commentary on contemporary infection prevention issues. Infect Control Hosp Epidemiol 2015;36:1073-1088.

7. Mody L, Foxman B, Bradley S, et al. Longitudinal assessment of multidrug-resistant organisms in newly admitted nursing facility patients: implications for an evolving population. Clin Infect Dis 2018;67:837-844.

8. Cassone M, Mantey J, Perri MB, et al. Environmental panels as a proxy for nursing facility patients with MRSA and VRE colonization. Clin Infect Dis 2018;67:861-868.

9. Lawton MP, Brody EM. Assessment of older people: self-maintaining and instrumental activities of daily living. Gerontologist 1969;9:179-186.

10. Larocque M, Carver S, Bertrand A, McGeer A, McLeod S, Borgundvaag B. Acquisition of bacteria on healthcare workers' hands after contact with patient privacy curtains. Am J Infect Control 2016;44:1385-1386.

\title{
Wither proper evaluation of falls for coexisting systemic infections?
}

\author{
Farrin A. Manian MD, MPH ${ }^{1}$ and Marine Lipartia $\mathrm{MD}^{2}$ \\ ${ }^{1}$ Department of Medicine, Mercy-St Louis, St Louis, Missouri and ${ }^{2}$ NUMEO Medical, Lexington, Massachusetts
}

To the Editor-We read the Society for Healthcare Epidemiology of America (SHEA) document on the reliability of nonlocalizing signs and symptoms as potential indicators of the presence of infection in nursing-home residents ${ }^{1}$ with great interest. The authors should be commended for their work aimed at curbing the overuse of antibiotics by highlighting the problem of frequent overdiagnosis of bacterial infections in long-term care facilities based on nonlocalizing signs and symptoms. We believe, however, that the section addressing falls, a common presentation in the elderly, ${ }^{2}$ deserves further discussion.

\footnotetext{
Author for correspondence: Farrin A. Manian, E-mail: Farrinman@gmail.com

Cite this article: Manian FA and Lipartia M. (2022). Wither proper evaluation of falls for coexisting systemic infections?. Infection Control \& Hospital Epidemiology, 43: 668-669, https://doi.org/10.1017/ice.2021.56
}

More specifically, the authors "do not recommend that clinicians evaluate a resident who has experienced a fall for the presence of infection." Visibly absent in the statement was a qualifier, such as in the absence of additional signs and symptoms of infection or "in isolation," terms used when also recommending against prompt evaluation for infection in other nonspecific symptoms (eg, behavioral changes exclusive of delirium, functional decline, or anorexia). The authors' argument against evaluation of falls as a potential sign of an underlying infection seems to revolve primarily around their concern over providers' overdiagnosis of urinary tract infections (UTIs) in a patient population at high risk of asymptomatic bacteriuria, which may in turn lead to unnecessary use of antibiotics for presumed urinary tract infection (UTI). They also add that their literature search failed to identify studies evaluating the association of falls with other infectious syndromes, 
including pneumonia. We wish to respectfully call attention to 3 other published studies ${ }^{3-5}$ addressing coexisting systemic infections (CSIs) in patients who fall, 2 of which seem to have been overlooked by the authors in their literature review. ${ }^{3,5}$

In 2017, we published data on 161 hospitalized patients $(75 \%$ aged $\geq 70$ years) who presented with falls and were diagnosed with a CSI. ${ }^{3}$ Although UTI was the most common CSI, it accounted for less than one-half $(44.1 \%)$ of all cases. Even in those with the diagnosis of UTI, nearly one-third had concurrent bacteremia, providing clear evidence that at least in some patients, the bacteriuria was not asymptomatic. Lower respiratory tract infection, sepsis of unclear source, and endocarditis led the list of CSIs outside the urinary tract. Interestingly, only $20 \%$ of patients with a fall and a CSI had fever or abnormally low temperatures on presentation; most (56\%) failed to meet the criteria for systemic inflammatory response syndrome (SIRS). CSI was initially unsuspected by providers in nearly $40 \%$ of cases.

In a more comprehensive study published in 2020 (subsequent to the literature review period specified in the document) involving 1,456 hospitalized patients (mean age, 72 years) presenting with a fall, CSI was diagnosed in $20.8 \%$ of patients and was more prevalent $(26.2 \%)$ among those aged $\geq 75$ years. ${ }^{4}$ CSI not related to UTI accounted for nearly one-half (45\%) of all cases, with pneumonia, skin and soft-tissue infections, and influenza/influenza-like illness leading the list. Several patient factors (eg, preexisting symptoms, presence of SIRS, confusion, age 50 years or older, inability to get up on own, and absence of fracture after a fall) were independently associated with the presence of CSI. Compared to those without CSI, fall patients with CSI had longer mean hospital stay (8.8 days vs 6.5 days; $P<.0001)$ and higher in-hospital mortality $(6.9 \%$ vs 3.8\%; OR, 1.9; 95\% CI, 1.1-3.3). More specifically, pneumonia and bacteremia were independently associated with in-hospital mortality (OR, 3.5 [95\% CI, 1.4-8.5] and OR 7.9 [95\% CI, 1.7$37.0]$, respectively). Again, CSI was not initially suspected by providers in a significant proportion $(32.5 \%)$ of cases.

Collectively, our data suggest that CSIs, including many not related to UTIs, are not uncommon among older patients presenting with a fall, often lack typical signs and symptoms associated with infections, are frequently overlooked by providers on initial encounter, and may be associated with a poorer outcome. These findings highlight the importance of timely diagnosis of infections in this patient population.

Notably, a 2010 study of patients with dementia on a psychogeriatric ward (mean age 80 years) reported that 1 of 5 falls were thought to have been precipitated by infections. ${ }^{5}$ In this study, $27 \%$ of infections did not involve the urinary tract; some patients had been diagnosed with pneumonia.

Physiologically, falls associated with infections (particularly when severe) may be explained by the impairment of skeletal (including quadriceps) function through a variety of mechanisms, including inflammatory cytokines and hypophosphatemia. ${ }^{6}$
Indeed, one can argue that falls may be a forme fruste of a serious infection in the elderly.

Admittedly, the scope and nature of CSIs in fall patients included in the epidemiological studies cited here may not necessarily coincide with those of nursing-home residents who fall without seeking medical care, do not require hospitalization, or do not have dementia. Nevertheless, nearly one-third of patients with falls seen in the emergency department may be hospitalized ${ }^{7}$ and nursing-home residents frequently suffer from dementia. ${ }^{8}$ Thus, we fear that at least some patients for whom evaluation for infection is not recommended by the current SHEA document may well have a CSI that would benefit from earlier diagnosis and treatment. The challenge, of course, is to provide clinicians with reliable tools that can (after careful examination of relevant patient symptoms and other factors) help them identify which patients should undergo evaluation for infection and which may be monitored. Further studies are clearly needed to advance the science of falls and infections. In the meantime, the recommendation to not evaluate nursing-home residents who suffer a fall for infection deserves further consideration.

\section{Acknowledgments.}

Financial support. No financial support was provided relevant to this article.

Conflicts of interest. All authors report no conflicts of interest relevant to this article.

\section{References}

1. Rowe TA, Jump RLP, Andersen BM, et al. Reliability of nonlocalizing signs and symptoms as indicators of the presence of infection in nursing-home residents. Infect Control Hosp Epidemiol 2020. doi: 10.1017/ice.2020.1282.

2. Ambrose AC, Paul G, Hasudorff JM. Risk factors for falls among older adults: a review of the literature. Maturitas 2013:75:51-61.

3. Blair A, Manian FA. Coexisting systemic infections in patients who present with a fall. Am J Med Sci 2017;353:22-26.

4. Manian FA, Hsu F, Huang D, et al. Coexisting systemic infections in patients hospitalized because of a fall: prevalence and risk factors. J Emerg Med 2020;58:733-740.

5. Tängman S, Eriksson S, Gustafson Y, et al. Precipitating factors for falls among patients with dementia on a psychogeriatric ward. Int Psychogeriatr 2010;22:641-649.

6. Guyan-Ramirez G, Decramer M. Mechanisms of striated muscle dysfunction during acute exacerbations of COPD. J Appl Physiol 2013;114: 1291-1299.

7. Shankar KN, Liu SW, Ganz DA. Trends and characteristics of emergency departments visits for fall-related injures on older adults, 2003-2010. West J Emerg Med 2017;18:785-793.

8. Bjork S, Juthberg C, Lindkvist M, et al. Exploring the prevalence and variance of cognitive impairment, pain, neuropsychiatric syndrome and ADL dependency among persons living in nursing homes; a cross-sectional study. BMC Geriatr 2016;16:154. 